\title{
Rapid and Sustained Effect of Dupilumab on Work Productivity in Patients with Difficult-to-treat Atopic Dermatitis: Results from the Dutch BioDay Registry
}

Lieneke F. M. ARIËNS ${ }^{1}$, Daphne S. BAKKER ${ }^{1}$, Lotte S. SPEKHORST ${ }^{1}$, Jorien VAN DER SCHAFT ${ }^{1}$, Judith L. THIJS ${ }^{1}$, Inge HAECK $^{2}$, Annebeth E. FLINTERMAN ${ }^{3}$, Marijke KAMSTEEG ${ }^{4}$, Marie L. A. SCHUTTELAAR ${ }^{5}$ and Marjolein S. DE BRUIN-WELLER ${ }^{1}$ ${ }^{1}$ National Expertise Center for Atopic Dermatitis, Department of Dermatology and Allergology, University Medical Center Utrecht, Departments of Dermatology, ${ }^{2}$ Reinier de Graaf Gasthuis, Delft, ${ }^{3}$ Diakonessenhuis, Utrecht, ${ }^{4}$ Radboud University Medical Center Nijmegen, and ${ }^{5}$ University Medical Center Groningen, University of Groningen, Groningen, The Netherlands

\begin{abstract}
Dupilumab treatment improves signs, symptoms, and quality of life in patients with moderate-to-severe atopic dermatitis. This study evaluated the impact of dupilumab treatment on absenteeism, presenteeism, and related costs in a large multi-centre cohort of adult patients with difficult-to-treat atopic dermatitis in daily practice. Patients treated with dupilumab participating in the Dutch BioDay Registry reporting employment were included. Absenteeism, presenteeism, and related costs at baseline and during follow-up were calculated using the Work Productivity and Activity Impairment questionnaire. A total of 218 adult patients with moderate-to-severe atopic dermatitis were included. Total work impairment reduced significantly from baseline $(35.5 \%)$ to week $52(11.5 \%)$, $p<0.001$. Median weekly productivity losses reduced significantly from baseline (C379.8 (140.7-780.8)) to week 52 (c0.0 (0.0-211.0), p<0.001). In this study, dupilumab treatment demonstrated a significant improvement in work productivity and reduction in associated costs in a large cohort of patients with difficultto-treat atopic dermatitis in daily practice.
\end{abstract}

Key words: atopic dermatitis; dupilumab; health-economics; absenteeism; presenteeism.

Accepted Aug 13, 2021; Epub ahead of print Aug 16, 2021

Acta Derm Venereol 2021; 101: adv00573.

Corr: Lieneke F. M. Ariëns, National Expertise Center for Atopic Dermatitis, Department of Dermatology and Allergology, University Medical Center Utrecht, NL-3584 CX Utrecht, The Netherlands. E-mail: I.f.m.ariens@ umcutrecht.nl

A topic dermatitis (AD) is one of the most common chronic inflammatory skin diseases worldwide and is characterized by intense pruritus and a relapsing and remitting course (1). Moderate-to-severe $\mathrm{AD}$ has a significant impact on the quality of life (QoL) of patients, due to its psychosocial impact, sleep loss, and concentration problems resulting from intense pruritus $(2,3)$. In addition, $\mathrm{AD}$ has a substantial economic burden caused by costs directly related to treatment and reduced work productivity $(3,4)$. A recent study on work productivity in patients with inadequately controlled AD demonstrated significantly higher absenteeism, presenteeism, and overall work impairment than in non-AD matched controls (5). In addition, costs due to productivity losses are a major contributor to the economic

\section{SIGNIFICANCE}

Real-life data on the effect of dupilumab treatment on absenteeism, presenteeism, and related costs in patients with atopic dermatitis treated in daily practice are lacking. In this study, the impact of dupilumab treatment on absenteeism, presenteeism, and related costs were evaluated in 128 patients with difficult-to-treat atopic dermatitis who were treated in daily practice. Rapid and sustained reductions in work absenteeism, presenteeism, and associated costs were observed in patients with moderate-to-severe atopic dermatitis treated with dupilumab in daily practice for 52 weeks.

burden of the disease in patients with moderate-to-severe $\mathrm{AD}$ indicated for systemic treatment (3).

Recent insight into the underlying immune pathogenesis of $\mathrm{AD}$ has led to the development of novel targeted therapies (6). Dupilumab, a fully monoclonal-antibody that targets the shared receptor component for interleukin (IL)-4 and IL-13, is the first biologic treatment for AD. The safety and effectiveness of dupilumab has been proven in phase III clinical trials and in daily practice (7-14). Dupilumab treatment significantly improves signs, symptoms, and QoL in patients with moderate-to-severe AD (7-14).

Real-life data on the effect of dupilumab treatment on absenteeism, presenteeism, and related costs in patients with AD treated in daily practice are lacking. Data derived from daily practice provides important information in addition to data from clinical trials, since there may be considerable differences in patient characteristics and treatment response. Patients participating in randomized controlled trials are screened by strict inclusion and exclusion criteria and treatment adherence, and the results might not be generalizable to a wider population in daily practice.

This prospective, real-life, registry study examined the impact of dupilumab treatment on absenteeism, presenteeism, and related costs in a large multi-centre cohort of adult patients with difficult-to-treat $\mathrm{AD}$.

\section{METHODS}

\section{Patient population}

Data were extracted from the BioDay Registry, a prospective multicentre observational longitudinal registry including all adult patients who started dupilumab for treatment-refractory $\mathrm{AD}$, ac- 
cording to the criteria established by the Dutch Society of Dermatology and Venereology (NVDV) (treatment $\geq 4$ months with $\geq 1$ conventional systemic therapy in an adequate dose). Patients included in the BioDay Registry were followed by 2 protocols. In the start-up phase of the BioDay Registry, patients were followed according to the "early access" protocol. Shortly after approval of dupilumab by the European Medicines Agency, dupilumab treatment was available only to patients included in the early access programme. These patients were intensively monitored by frequent follow-up visits. After market access of dupilumab, patients were followed according to the BioDay protocol, a simplified protocol with less frequent follow-up visits. Therefore, outcomes regarding work productivity and activity impairment were available at baseline and week 52 for both cohorts, at week 16 for patients included the early access cohort, and at week 28 for patients included in the BioDay cohort. The BioDay Registry was considered as non-interventional by the local medical ethics committee and collection of data was performed according to the principles of the Declaration of Helsinki. All patients provided written informed consent. ClinicalTrials.gov identifier: NCT03549416.

\section{Outcomes}

Patients included in this analysis were assessed at baseline, after 16 (early access protocol), 28 (BioDay protocol), and 52 weeks of dupilumab treatment. Patients who indicated active employment status at any of the assessment visits and with available scores on the Work Productivity and Activity Impairment questionnaire general health (WPAI-GH) at baseline and $\geq 1$ of the follow-up visits were included in this analysis.

The WPAI questionnaire is a validated, self-administered instrument to measure impairments in work and activities across 4 domains in the past 7 days; 1 : absenteeism or percentage work time missed due to health problems; 2 : presenteeism or percentage impairment while working due to ill health; 3: percentage of overall work impairment (absenteeism + presenteeism); and 4: percentage of activity impairment due to the health problem (15). The WPAI questionnaire used in this study was translated in Dutch by the Mapi Research Trust on behalf of Reilly Associates. The Dutch version of the WPAI-GH itself is not validated. Disease severity at baseline, week 16 , week 28 , and week 52 were asses- sed by the Eczema Area and Severity Index (EASI: 0-72) (16). Patient-reported outcomes, including the Patient-Oriented Eczema Measure (POEM: 0-28), weekly mean numerical rating scale (NRS: 0-10), and pruritus and Dermatology Life Quality Index (DLQI: 0-30) were reported at baseline, week 16, week 28, and week 52 (17-19). In the start-up phase of the BioDay Registry, the effect of dupilumab on concomitant allergic diseases was not monitored by using validated questionnaires. Therefore, these data are not available for the current cohort of patients.

\section{Analysis}

Costs of productivity losses were calculated according to the Dutch guideline for economic evaluations in healthcare (20). Costs of productivity loss included costs due to productivity losses from being absent from work (absenteeism) and being less productive at work (presenteeism). Total productivity losses for employed patients were calculated by hours of productivity losses (hours of absenteeism + presenteeism) multiplied by the value of productivity loss per hour. Outcome measures at different follow-up visits were compared with baseline using the Wilcoxon signed-rank test. Missing data during follow-up were imputed by last observation carried forward (LOCF) method. Statistical analyses were conducted using SPSS (for Windows, version 25.0, SPSS Inc.) and Prism (version 7.4; $120 \mathrm{GraphPad}$ ).

\section{RESULTS}

In total, 218 patients with moderate-to-severe $\mathrm{AD}$ were included (mean (interquartile range (IQR) age 39.0 years (28.5-51.9); 139 patients (65.0\%) were male) (Table I). Out of 218 patients, $99(45.4 \%)$ had a history of $\geq 2$ oral immunosuppressive treatments. At baseline, median (IQR) baseline EASI score was 18.1 (12.1-26.3). Patients reported a median (IQR) baseline POEM score of 22.0 (18.0-26.0), a median (IQR) baseline weekly mean pruritus NRS score of 7.0 (6.0-8.0) and a median (IQR) baseline DLQI score of 12.0 (8.0-18.0).

Table I. Baseline characteristics

\begin{tabular}{|c|c|c|c|c|}
\hline Characteristics & Total group $(n=218)$ & $\begin{array}{l}\text { Early access protocol } \\
(n=134)\end{array}$ & $\begin{array}{l}\text { BioDay protocol } \\
(n=84)\end{array}$ & $p$-value \\
\hline Age, years, median (IQR) & $39.0(28.5-51.9)$ & $42.6(29.4-53.9)$ & $33.6(28.3-48.7)$ & 0.035 \\
\hline Male sex, $n(\%)$ & $139(65.0)$ & $84(62.7)$ & $55(65.5)$ & 0.608 \\
\hline \multicolumn{5}{|l|}{ Atopic/allergic diseases at baseline, $n(\%)$} \\
\hline Allergic rhinitis & $152(71.4)$ & $92(69.7)$ & $60(74.0)$ & \multirow[t]{2}{*}{0.352} \\
\hline Missing & $5(2.3)$ & $2(1.5)$ & $3(3.6)$ & \\
\hline Asthma & $126(59.2)$ & $82(62.1)$ & $44(54.3)$ & \multirow[t]{2}{*}{0.237} \\
\hline Missing & $5(2.3)$ & $2(1.5)$ & $3(3.6)$ & \\
\hline Food allergy & $125(58.7)$ & $66(50.0)$ & $37(45.7)$ & \multirow[t]{2}{*}{0.167} \\
\hline Missing & $5(2.3)$ & $2(1.5)$ & $3(3.6)$ & \\
\hline Allergic conjunctivitis & $103(48.4)$ & $79(60.3)$ & $46(56.8)$ & \multirow[t]{2}{*}{0.830} \\
\hline Missing & $5(2.3)$ & $3(2.2)$ & $3(3.6)$ & \\
\hline History of $\geq 2$ oral immunosuppressive treatments, $n(\%)$ & $99(45.4)$ & $65(48.5)$ & $34(40.4)$ & 0.290 \\
\hline Previous use of cyclosporin $\mathrm{A}, n(\%)$ & $205(94.0)$ & $125(93.3)$ & $80(95.2)$ & 0.553 \\
\hline Previous use of methotrexate, $n(\%)$ & $56(25.7)$ & $39(29.1)$ & $17(20.2)$ & 0.145 \\
\hline Previous use of azathioprine, $n(\%)$ & $45(20.6)$ & $30(22.4)$ & $25(29.8)$ & 0.421 \\
\hline Previous use of mycophenolate mofetil/enteric-coated mycophenolate sodium, $n(\%)$ & $41(18.8)$ & $29(21.6)$ & $11(13.1)$ & 0.113 \\
\hline Eczema Area and Severity Index score, median (IQR) & $18.1(12.1-26.3)$ & $18.9(12.5-27.2)$ & $17.0(11.1-24.7)$ & 0.325 \\
\hline \multicolumn{5}{|l|}{ IGA score, $n(\%)$} \\
\hline 3 (moderate) & $93(43.1)$ & $58(43.3)$ & $35(42.7)$ & \multirow[t]{2}{*}{ na } \\
\hline 4 (severe) & $52(24.1)$ & $56(41.8)$ & $32(39.0)$ & \\
\hline Weekly mean pruritus NRS score, median (IQR) & $7.0(6.0-8.0)$ & $7(6.0-8.0)$ & $7.0(5.0-8.0)$ & 0.643 \\
\hline Patient-Oriented Eczema Measure score, median (IQR) & $22.0(18.0-26.0)$ & $23.0(18.0-27.0)$ & $20.0(17.5-25.0)$ & 0.007 \\
\hline Dermatology Life Quality Index score, median (IQR) & $12.0(8.0-18.0)$ & $13.0(8.0-18.8)$ & $12.0(7.5-17.0)$ & 0.393 \\
\hline
\end{tabular}

IQR: interquartile range; NRS: numerical rating scale. 
Table II. Impairment in weekly productivity, activity and disease severity

\begin{tabular}{|c|c|c|c|c|c|c|c|c|}
\hline & \multicolumn{3}{|l|}{ Baseline } & \multirow{2}{*}{$\begin{array}{l}\text { Week } 16 \\
\\
\text { Early access } \\
\text { protocol }(n=134)\end{array}$} & \multirow{2}{*}{$\begin{array}{l}\text { Week } 28 \\
\begin{array}{l}\text { BioDay protocol } \\
(n=84)\end{array}\end{array}$} & \multicolumn{3}{|l|}{ Week 52} \\
\hline & $\begin{array}{l}\text { Total group } \\
(n=218)\end{array}$ & $\begin{array}{l}\text { Early access } \\
\text { protocol } \\
(n=134)\end{array}$ & $\begin{array}{l}\text { BioDay } \\
\text { protocol } \\
(n=84)\end{array}$ & & & $\begin{array}{l}\text { Total group } \\
(n=218)\end{array}$ & $\begin{array}{l}\text { Early access } \\
\text { protocol } \\
(n=134)\end{array}$ & $\begin{array}{l}\text { BioDay protocol } \\
(n=84)\end{array}$ \\
\hline Absenteeism, \%, mean (SD) & $17.2(31.5)$ & $20.8(34.4)$ & $11.6(25.7)$ & $7.6(23.3)^{* * *}$ & $3.4(11.4)^{*}$ & $4.0(15.5)^{* * *}$ & $2.7(15.0)^{* * *}$ & $4.5(16.4)^{*}$ \\
\hline Presenteeism, \%, mean (SD) & $35.2(28.2)$ & $36.2(27.2)$ & $33.9(29.6)$ & $15.7(23.4)^{* * *}$ & $13.2(21.7)^{* * *}$ & $11.8(18.9)^{* * *}$ & $13.3(20.0)^{* * *}$ & $9.4(16.9)^{* * *}$ \\
\hline $\begin{array}{l}\text { Overall work impairment, \%, } \\
\text { mean (SD) }\end{array}$ & $35.5(28.5)$ & $36.0(27.3)$ & $34.8(30.4)$ & $15.9(25.4)^{* * *}$ & $13.8(22.8)^{* * *}$ & $11.5(18.4)^{* * *}$ & $12.7(19.3)^{* * *}$ & $9.5(16.9) * * *$ \\
\hline $\begin{array}{l}\text { Activity impairment, } \% \text {, mean } \\
\text { (SD) }\end{array}$ & $43.4(28.6)$ & $44.8(29.7)$ & $40.7(26.3)$ & $17.5(23.7)^{* * *}$ & $22.4(21.2)^{* * *}$ & $16.9(21.9)^{* * *}$ & $15.4(22.4)^{* * *}$ & $20.8(20.5)^{* * *}$ \\
\hline $\begin{array}{l}\text { Total EASI score, median } \\
\text { (IQR) }\end{array}$ & $\begin{array}{l}18.1(12.1- \\
26.3)\end{array}$ & $\begin{array}{l}18.9(12.5- \\
27.2)\end{array}$ & $\begin{array}{l}17.0(11.1- \\
24.7)\end{array}$ & $3.6(1.6-7.0)^{* * *}$ & $3.6(1.4-6.9)^{* * *}$ & $2.7(1.2-5.0)^{* * * *}$ & $2.4(1.4-5.0)^{* * *}$ & $2.8(1.1-4.9)^{* * *}$ \\
\hline $\begin{array}{l}\text { Weekly mean pruritus NRS } \\
\text { score, median (IQR) }\end{array}$ & $7.0(6.0-8.0)$ & $7(6.0-8.0)$ & $7.0(5.0-8.0)$ & $3.0(1.0-5.0) * * *$ & $3.0(1.0-5.0)^{* * *}$ & $2.0(1.0-4.0)^{* * *}$ & $2.0(1.0-4.0) * * *$ & $2.0(1.0-4.0)^{* * *}$ \\
\hline POEM score, median (IQR) & $\begin{array}{l}22.0(18.0- \\
26.0)\end{array}$ & $\begin{array}{l}23.0(18.0- \\
27.0)\end{array}$ & $\begin{array}{l}20.0(17.5- \\
25.0)\end{array}$ & $8.0(3.0-13.0)^{* * *}$ & $7.0(4.0-10.0)^{* * *}$ & $6.0(3.0-11.0)^{* * *}$ & $6.0(3.0-11.0)^{* * *}$ & $6.0(3.0-10.5)^{* * *}$ \\
\hline DLQI score, median (IQR) & $\begin{array}{l}12.0 \\
(8.0-18.0)\end{array}$ & $\begin{array}{l}13.0 \\
(8.0-18.8)\end{array}$ & $\begin{array}{l}12.0(7.5- \\
17.0)\end{array}$ & $2.0(1.0-5.0)^{* * *}$ & $2.0(1.0-4.5)^{* * *}$ & $2.0(1.0-3.0) * * *$ & $2.0(1.0-3.0)^{* * *}$ & $2.0(1.0-3.5)^{* * *}$ \\
\hline
\end{tabular}

Data were analysed using a Wilcoxon matched-pairs signed-rank test.

${ }^{*} p<0.05, * * p<0.01, * * * p<0.001$ to baseline.

SD: standard deviation; EASI: Eczema Area and Severity Index; IQR: interquartile range; NRS: numerical rating scale; POEM: Patient-Oriented Eczema Measure; DLQI: Dermatology Life Quality Index.

Out of 218 patients, $134(61.5 \%)$ were followed according the early access protocol and $84(38.5 \%)$ were followed according the BioDay protocol. Patients included in the early access cohort were significantly older (median (IQR) 42.6 years (29.4-53.9)) compared with patients included in the BioDay cohort (median (IQR) 33.6 years $(28.3-48.7))(p=0.035)$. The baseline POEM score was significantly higher in the early access cohort (median (IQR) 23.0 (18.0-27.0)) compared with the BioDay cohort $(20.0(17.5-25.0))(p=0.007)$. Other baseline characteristics were similar among the groups.

Dupilumab treatment resulted in a significant reduction in AD signs, symptoms, and impact on quality of life from baseline to week 4 , week 16, week 28, and improvement sustained until week 52 (data not reported). In the early access cohort, mean percentage absenteeism reduced significantly from baseline (20.8\% (SD 34.4)) to week 16 (7.6\% (SD 23.3), $p<0.001)$ and to week 52 (2.7\% (SD 15.0), $p<0.001$ ) (Table II). In the BioDay cohort, mean absenteeism reduced significantly from baseline (11.6\% (SD 25.7)) to week 28 (3.4\% (SD 11.4), $p=0.008)$ and to week 52 (4.5\% (SD 16.4), $p=0.05)$. In the total group of patients, absenteeism reduced significantly from baseline (17.2\% (SD 31.5)) to week 52 (4.5\% (SD 16.4), $p<0.001$ ) (Fig. 1). Mean presenteeism, overall work impairment (absenteeism + presenteeism) and activity impairment also reduced significantly from baseline to week $16(p<0.001)$ (early access cohort), from baseline to week $28(p<0.001)$ (BioDay cohort) and from baseline to week $52(p<0.001)$ in both cohorts (Fig. 1). Mean change over time in presenteeism and total work impairment, from baseline to week 52, was significantly higher in patients without self-reported asthma at baseline compared with patients with self-reported asthma at baseline (presenteeism: $-30.1 \%$ (SD 29.1) vs $-19.6 \%$ (SD 25.1) $(p=0.017)$; total work impairment: $-30.6 \%$ (SD 29.4) vs $-19.9 \%$ (SD 26.3) $(p=0.019))$. No other differences in change over time of WPAI scores from baseline to week 52 were observed among patients with other atopic comorbidities. Mean total weekly hours of productivity loss (hours absenteeism + hours presenteeism) significantly decreased from baseline (to week $16(p<0.001)$ (early access cohort), from baseline to week $28(p<0.001)$ (BioDay cohort) and from baseline to week $52(p<0.001)$ in both cohorts (Table III). Reduced productivity loss resulted in a significant decrease of related costs during dupilumab treatment compared with baseline. The weekly annual median costs (hours absenteeism + hours presenteeism * value of productivity loss per hour $(€ 35.17))$ decreased from baseline (€422.0 (IQR 140.7-747.4)) to week 16 (€0.0 (IQR 0.0-174.1), $p<0.001$ ) and to week $52(€ 0.0$ (IQR $0.0-211.0), p<0.001)$ in the early access cohort. In the BioDay cohort, median costs decreased from baseline ( $€ 281.4$ (IQR 126.6-844.1)) to week 28 $(€ 0.0$ (IQR 0.0-225.1), $p<0.001)$ and to week $52(€ 0.0$ (IQR $0.0-128.4), p<0.001$ ). In the total group of patients, median weekly costs decreased from baseline $(€ 379.8$ (IQR 140.7-780.8)) to week 52 (€0.0 (IQR 0.0-211.0)).

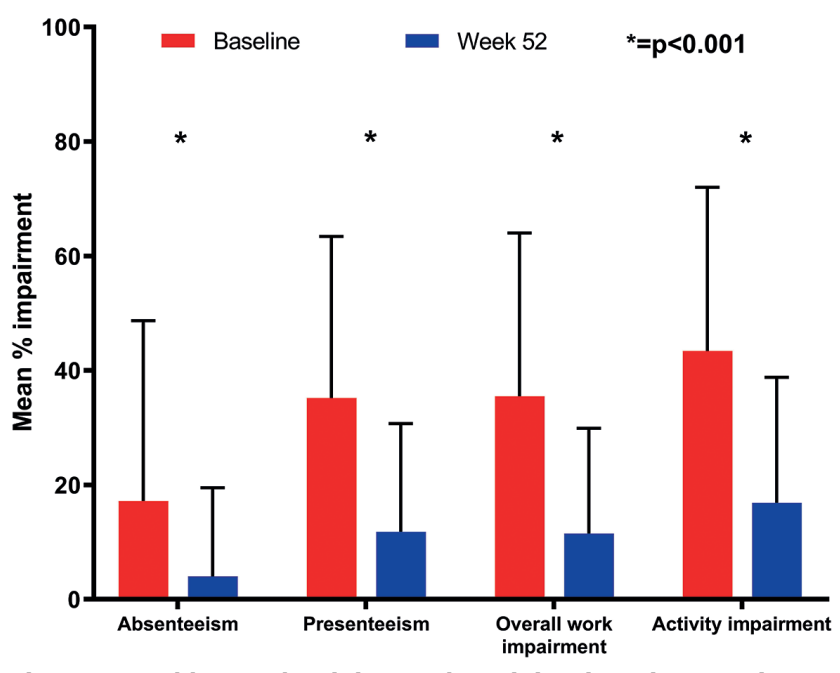

Fig. 1. Weekly productivity and activity impairment (mean percentage (standard deviation)) at baseline and after 52 weeks of treatment with dupilumab. 
Table III. Total weekly hours of productivity loss and related costs

\begin{tabular}{|c|c|c|c|c|c|c|c|c|}
\hline & \multicolumn{3}{|l|}{ Baseline } & \multirow{2}{*}{$\begin{array}{l}\text { Week } 16 \\
\text { Early access } \\
\text { protocol }(n=134)\end{array}$} & \multirow{2}{*}{$\begin{array}{l}\text { Week } 28 \\
\begin{array}{l}\text { BioDay protocol } \\
(n=84)\end{array}\end{array}$} & \multicolumn{3}{|l|}{ Week 52} \\
\hline & $\begin{array}{l}\text { Total group } \\
(n=218)\end{array}$ & $\begin{array}{l}\text { Early access } \\
\text { protocol } \\
(n=134)\end{array}$ & $\begin{array}{l}\text { BioDay } \\
\text { protocol } \\
(n=84)\end{array}$ & & & $\begin{array}{l}\text { Total group } \\
(n=218)\end{array}$ & $\begin{array}{l}\text { Early access } \\
\text { protocol }(n=134)\end{array}$ & $\begin{array}{l}\text { BioDay protocol } \\
(n=84)\end{array}$ \\
\hline $\begin{array}{l}\text { Absenteeism + } \\
\text { presenteeism (h/week), } \\
\text { mean (SD) }\end{array}$ & $13.7(12.2)$ & $14.1(12.2)$ & $12.9(12.2)$ & $4.4(8.0)^{* * *}$ & $5.4(9.5)^{* * *}$ & $4.7(9.3)^{* * *}$ & $4.4(8.8)^{* * *}$ & $5.1(10.2)^{* * *}$ \\
\hline $\begin{array}{l}\text { Weekly productivity losses } \\
(€) \text {, median (IQR) }\end{array}$ & $\begin{array}{l}379.8(140.7- \\
780.8)\end{array}$ & $\begin{array}{l}422.0(140.7- \\
747.4)\end{array}$ & $\begin{array}{l}281.4(126.6- \\
844.1)\end{array}$ & $0.0(0.0-174.1)^{* * *}$ & $0.0(0.0-225.1)^{* * *}$ & $0.0(0.0-211.0)^{* * *}$ & $0.0(0.0-211.0)^{* * *}$ & $0.0(0.0-128.4)^{* * *}$ \\
\hline
\end{tabular}

SD: standard deviation; IQR: interquartile range.

Estimated extrapolated median yearly costs due to productivity losses significantly decreased from baseline (€19751.5 (IQR 7315.4-40966.0) to week 52 (€0.0 (IQR $0.0-10973.0), p<0.001)$ in the total group of patients.

\section{DISCUSSION}

This study demonstrated a rapid and sustained reduction in work absenteeism, presenteeism, and associated costs in patients with moderate-to-severe $\mathrm{AD}$ treated with dupilumab in daily practice.

In a previous study by our group, we demonstrated a substantial economic burden in patients with $\mathrm{AD}$ indicated for systemic treatment (3). In this study, the mean (SD) reported absenteeism over the past 7 days at baseline was $15.7 \%$, mean reported presenteeism was $26.4 \%$, and overall work impairment due to health was $28.2 \%$, compared with $17.2 \%, 35.2 \%$, and $35.5 \%$, respectively, at baseline in the current study. The slightly higher percentages in the current study could possibly be explained by the fact that the study population had a more severe form of $\mathrm{AD}$; all patients included in this study had very difficult-to-treat $\mathrm{AD}$ and had failed multiple treatments with oral immunosuppressive drugs.

In our previous study, costs due to productivity losses were the major contributor to the economic burden in this group of patients. Costs of productivity loss were $€ 10,040$ $(€ 6,260-14,012)$ per patient year (PPY) for the total group, $€ 6,886(€ 4,188-10,129)$ PPY for patients with controlled $A D$ vs $€ 13,702(€ 6,124-22,996)$ for patients with uncontrolled $\mathrm{AD}$. In the present study, estimated extrapolated median yearly costs at baseline due to productivity losses were higher ( $€ 19751.5$ (IQR 7315.4-40966.0)) compared with our previous study which may be explained by the inclusion of a more severely afflicted population, which is also reflected by higher baseline disease severity scores. The estimated extrapolated median yearly costs due to productivity losses significantly decreased to $€ 0.0$ ((IQR $0.0-10,973.0), p<0.001)$ after 52 weeks of treatment with dupilumab. Given the higher price of dupilumab treatment compared with conventional immunosuppressive treatments, direct costs related to treatment will substantially increase in patients treated with dupilumab. However, this increase in direct costs could be compensated by savings in costs due to productivity losses in patients treated with dupilumab.
Recently, pooled analysis of data from the SOLO 1 and 2 randomized, controlled clinical trials, demonstrated significant reduction in work/school absenteeism and related costs in patients with moderate-to-severe AD treated with dupilumab compared with placebo (21). However, this study did not include the impact of dupilumab on presenteeism and associated costs, which has been demonstrated to be a major contributor to the economic burden of the disease. Therefore, the results of the current study on the impact of dupilumab on absenteeism, presenteeism, and associated costs in patients treated with dupilumab in daily practice provide important additional information.

The current study should be interpreted in the context of several limitations. Patients included in this study were followed by 2 different protocols. Therefore, it was not possible to perform a pooled analysis including all patients at all follow-up visits. In addition, shortly after approval of dupilumab by the European Medicines Agency, dupilumab treatment was available only for patients included in the early access programme. Patients were indicated for treatment with dupilumab in the early access programme in cases of severe $\mathrm{AD}$ with limited alternative treatment options. Therefore, this cohort may represent a more severe population compared with patients included in the BioDay cohort, which could explain the higher baseline productivity losses in the early access cohort compared with the BioDay cohort. Another limitation of this study is the use of the WPAI-GH to calculate work impairment and related costs. The WPAI-GH is a non-disease-specific tool, and observed decreases in total work impairment could also be the effect of other diseases (e.g. impact of dupilumab on allergic comorbidities). In this study, we observed a significantly higher change over time, we observed in presenteeism and total work impairment from baseline to week 52 in patients without self-reported asthma, compared with patients with self-reported asthma at baseline. These data suggest that WPAI scores are also affected by the presence of allergic comorbidities, including asthma. Dupilumab has shown to improve signs and symptoms, reduce exacerbations and reduce the amount of oral corticosteroids in patients with moderate-to-severe eosinophilic or oral steroid dependent asthma (22-24). In this study, the severity of asthma and whether patients had eosinophilic or oral steroid dependent asthma was unknown. Therefore, the effect of dupilumab on concomitant asthma might be less significant compared with the effect 
on $\mathrm{AD}$ in this severe AD population. Patients with selfreported asthma at baseline might still experience signs and symptoms of active asthma at week 52, which may explain the lower change over time in WPAI outcomes.

The absence of an analysis of the direct costs is another limitation of this study. Since patients were included shortly after approval of dupilumab by the European Medicines Agency (early access cohort) and market access of dupilumab (BioDay cohort), and were intensively monitored by frequent follow-up visits and laboratory monitoring, analysis of the direct costs would not have reflected a real-life setting, and would have resulted in an overestimation of direct costs. However, given the high cost of dupilumab treatment compared with conventional immunosuppressive treatments, it is likely that direct costs related to treatment may be substantially higher in patients treated with dupilumab. Future research should further investigate the effect of dupilumab treatment on direct as well as indirect costs.

In conclusion, patients with difficult-to-treat AD reporting employment demonstrated significant, rapid and sustained reductions in absenteeism, presenteeism, total work impairment and activity impairment. In addition, indirect costs due to productivity losses were significantly reduced. Future research should further investigate the direct as well as indirect costs involved in patient treatment with dupilumab and other new treatment options in atopic dermatitis in daily practice.

\section{REFERENCES}

1. Weidinger S, Novak N. Atopic dermatitis. Lancet 2016; 387: 1109-1122.

2. Drucker AM, Wang AR, Li WQ, Sevetson E, Block JK, Qureshi AA. The burden of atopic dermatitis: summary of a report for the National Eczema Association. J Invest Dermatol 2017; 137: 26-30.

3. Ariens LFM, van Nimwegen KJM, Shams M, de Bruin DT, van der Schaft J, van Os-Medendorp $\mathrm{H}$, et al. Economic burden of adult patients with moderate to severe atopic dermatitis indicated for systemic treatment. Acta Derm Venereol 2019; 99: 762-768.

4. Eckert L, Gupta S, Amand C, Gadkari A, Mahajan P, Gelfand JM. The burden of atopic dermatitis in US adults: health care resource utilization data from the 2013 National Health and Wellness Survey. J Am Acad Dermatol 2018; 78: 54-61.

5. Eckert L, Gupta S, Amand C, Gadkari A, Mahajan P, Gelfand JM. Impact of atopic dermatitis on health-related quality of life and productivity in adults in the United States: an analysis using the National Health and Wellness Survey. J Am Acad Dermatol 2017; 77: 274-279.

6. Paller AS, Kabashima K, Bieber T. Therapeutic pipeline for atopic dermatitis: end of the drought? J Allergy Clin Immun 2017; 140: 633-643.

7. Blauvelt A, de Bruin-Weller M, Gooderham M, Cather JC, Weisman J, Pariser D, et al. Long-term management of moderate-to-severe atopic dermatitis with dupilumab and concomitant topical corticosteroids (LIBERTY AD CHRONOS): a 1-year, randomised, double-blinded, placebo-controlled, phase 3 trial. Lancet 2017; 389: 2287-2303.

8. Simpson EL, Bieber T, Guttman-Yassky E, Beck LA, Blauvelt A, Cork MJ, et al. Two phase 3 trials of dupilumab versus placebo in atopic dermatitis. N Engl J Med 2016; 375: 2335-2348.

9. de Bruin-Weller M, Thaçi D, Smith CH, Reich K, Cork M, Radin
A, et al. Dupilumab with concomitant topical corticosteroids in adult patients with atopic dermatitis who are not adequately controlled with or are intolerant to ciclosporin A, or when this treatment is medically inadvisable: a placebo-controlled, randomized phase 3 clinical trial (LIBERTY AD CAFÉ). Br J Dermatol 2018; 178: 1083-1101.

10. Ariens LF, van der Schaft J, Bakker DS, Balak D, Romeijn ML, Kouwenhoven $T$, et al. Dupilumab is very effective in a large cohort of difficult-to-treat adult atopic dermatitis patients: first clinical and biomarker results from the BioDay registry. Allergy 2020; 75: 116-126.

11. de Wijs LEM, Bosma AL, Erler NS, Hollestein LM, Gerbens LAA, Middelkamp-Hup MA, et al. Effectiveness of dupilumab treatment in 95 patients with atopic dermatitis: daily practice data. Br J Dermatol 2020; 182: 418-426.

12. Faiz S, Giovannelli J, Podevin C, Jachiet M, Bouaziz JD, Reguiai $Z$, et al. Effectiveness and safety of dupilumab for the treatment of atopic dermatitis in a real-life French multicenter adult cohort. J Am Acad Dermatol 2019; 81: 143-151.

13. Bosma AL, de Wijs LEM, Hof $M H$, van Nieuwenhuizen $B R$, Gerbens LAA, Middelkamp-Hup MA, et al. Long-term effectiveness and safety of treatment with dupilumab in patients with atopic dermatitis: results of the TREAT NL (TREatment of ATopic eczema, the Netherlands) registry. J Am Acad Dermatol 2020; 83: 1375-1384.

14. Ariens LF, van der Schaft J, Spekhorst LS, Bakker DS, Romeijn GLE, Kouwenhoven TA, et al. Dupilumab shows long-term effectiveness in a large cohort of treatment-refractory atopic dermatitis patients in daily practice: 52 -weeks results from the Dutch BioDay registry. J Am Acad Dermatol 2021; 84: 1000-1009.

15. Reilly MC, Zbrozek AS, Dukes EM. The validity and reproducibility of a work productivity and activity impairment instrument. Pharmacoeconomics 1993; 4: 353-365.

16. Hanifin JM, Thurston M, Omoto M, Cherill R, Tofte SJ, Graeber M. The eczema area and severity index (EASI): assessment of reliability in atopic dermatitis. EASI Evaluator Group. Exp Dermatol 2001; 10: 11-18.

17. Charman CR, Venn AJ, Ravenscroft JC, Williams HC. Translating Patient-Oriented Eczema Measure (POEM) scores into clinical practice by suggesting severity strata derived using anchor-based methods. Br J Dermatol 2013; 169: 1326-1332.

18. Yosipovitch G, Reaney M, Mastey V, Eckert L, Abbe A, Nelson $L$, et al. Peak Pruritus Numerical Rating Scale: psychometric validation and responder definition for assessing itch in moderate-to-severe atopic dermatitis. $\mathrm{Br}$ J Dermatol 2019; 181: 761-769.

19. Finlay AY, Khan GK. Dermatology Life Quality Index (DLQI) a simple practical measure for routine clinical use. Clin Exp Dermatol 1994; 19: 210-216.

20. Zorginstituut N. Richtlijn voor het uitvoeren van economische evaluaties in de gezondheidszorg. 2016. Available from: https://www.zorginstituutnederland.nl/publicaties/ publicatie/2016/02/29/richtlijn-voor-het-uitvoeren-van-economische-evaluaties-in-de-gezondheidszorg (English version is available from: https://english.zorginstituutnederland.nl/ publications/reports/2016/06/16/guideline-for-economicevaluations-in-healthcare).

21. de Bruin-Weller M, Simpson EL, Cork M, Chen Z, Msihid J, Taniou $C$, et al. Dupilumab reduces absenteeism in patients with moderate to severe atopic dermatitis: Pooled results from the LIBERTY AD SOLO clinical trials. J Am Acad Dermatol 2020; 83: 1499-1501.

22. Rabe KF, Nair P, Brusselle G, Maspero JF, Castro M, Sher L, et al. Efficacy and safety of dupilumab in glucocorticoid-dependent severe asthma. N Engl J Med 2018; 378: 2475-2485.

23. Castro M, Corren J, Pavord ID, Maspero J, Wenzel S, Rabe KF, et al. Dupilumab efficacy and safety in moderate-to-severe uncontrolled asthma. N Engl J Med 2018; 378: 2486-2496.

24. Busse WW, Maspero JF, Rabe KF, Papi A, Wenzel SE, Ford LB, et al. Liberty Asthma QUEST: phase 3 randomized, double-blind, placebo-controlled, parallel-group study to evaluate dupilumab efficacy/safety in patients with uncontrolled, moderateto-severe asthma. Adv Ther 2018; 35: 737-748. 